\title{
Chaos Anti-Synchronization between Chen System and Genesio System
}

\author{
Xianyong Wu, Hao Wu, Zhengrong Yan, Yuehua Huang \\ College of Electrical Engineering \& New Energy, China Three Gorges University, Yichang, China \\ Email: 420372724@qq.com
}

Received 27 June 2016; accepted 10 July 2016; published 14 July 2016

Copyright (C) 2016 by authors and OALib.

This work is licensed under the Creative Commons Attribution International License (CC BY). http://creativecommons.org/licenses/by/4.0/

CC) (i) Open Access

\begin{abstract}
In this paper, anti-synchronization of two different chaotic systems is investigated. On the basis of Lyapunov theory, adaptive control scheme is proposed when system parameters are unknown or uncertain; sufficient conditions for the stability of the error dynamics are derived, where the controllers are designed by using the sum of the state variables in chaotic systems. Numerical simulations are performed for the Chen system and Genesio system to demonstrate the effectiveness of the proposed control strategy.
\end{abstract}

\section{Keywords}

\author{
Chaotic System, Anti-Synchronization, Adaptive Control \\ Subject Areas: Chaotic System, Anti-Synchronization, Adaptive Control
}

\section{Introduction}

Since the pioneering work by Pecora and Carroll [1], chaos synchronization, a very active topic in nonlinear science, has received increasing attention. The concept of synchronization has been extended in scope, for example to generalized synchronization [2]-[4], phase synchronization [5], lag synchronization [6], and even anti-phase synchronization (APS) [7]-[9]. APS can also be interpreted as anti-synchronization (AS), which is a phenomenon in which the state variables of the synchronized systems have the same amplitude as but opposite signs to those of the driving system. Therefore, the sums of two signals are expected to converge to zero when AS appears. Recently, Singh and Roy generalized active control to AS for two systems [10]. However, it seems that there are fewer previous results on AS between two different systems with unknown parameters using adaptive control. In this paper, we will focus on the AS of two different systems. Adaptive control methods will be employed; a sufficient condition for anti-synchronization is derived rigorously. Numerical simulations on Chen system and Genesio system are performed, which demonstrate the effectiveness and feasibility of the proposed control technique. 
The layout of the rest of the paper is as follows. Section 2 describes the systems and their mathematical models; in Section 3, adaptive anti-synchronization between Chen and Genesio systems is presented; numerical simulation results are given for illustration and verification. Finally, conclusions are drawn in Section 4.

\section{Systems Description and Mathematical Models}

Consider nonlinear chaotic system as follows.

$$
\left\{\begin{array}{l}
\dot{x}=f(t, x) \\
\dot{y}=g(t, y)+u(t, x, y)
\end{array}\right.
$$

where $x, y \in R^{n}, f, g \in R \times R^{n} \rightarrow R^{n}$ are differentiable functions, the first equation in (1) is the drive system, and the second one is the response system, $u(t, x, y)$ is the control input. Let $\mathbf{e}=\mathbf{y}+\mathbf{x}$ be the anti-synchronization error, our goal is to design controllers $\mathbf{u}$ such that the trajectory of the response system with initial conditions $y_{0}$ can asymptotically approach the drive system with initial conditions $x_{0}$ reversely and implement anti-synchronization finally, in the sense that

$$
\lim _{t \rightarrow \infty}\|e\|=\lim _{t \rightarrow \infty}\left\|y\left(t, y_{0}\right)+x\left(t, x_{0}\right)\right\|=0
$$

where $\|\cdot\|$ is the Euclidean norm.

The Genesio system, proposed by Genesio and Tesi [11], is one of paradigms of chaos since it captures many features of chaotic systems. It includes a simple square part and three simple ordinary differential equations that depend on three negative real parameters. The dynamic equations of the system is given by

$$
\left\{\begin{array}{l}
\dot{x}=y \\
\dot{y}=z \\
\dot{z}=a x+b y+c z+x^{2}
\end{array}\right.
$$

where $x, y, z$ are state variables, when $a=-6, b=-2.92, c=-1.2$, system (2) is chaotic.

Chen system is described by

$$
\left\{\begin{array}{l}
\dot{x}_{1}=a_{1}\left(y_{1}-x_{1}\right) \\
\dot{y}_{1}=\left(c_{1}-a_{1}\right) x_{1}-x_{1} z_{1}+c_{1} y_{1} \\
\dot{z}_{1}=x_{1} y_{1}-b_{1} z_{1}
\end{array}\right.
$$

When $a_{1}=35, b_{1}=3, c_{1}=28$, system (3) is chaotic.

In the next section, we will study chaos anti-synchronization between Chen and Genesio systems with known or unknown parameters using adaptive control.

\section{Adaptive Anti-Synchronization between Chen and Genesio System with Unknown Parameters}

We assume that Genesio system (2) is the drive system, and the controlled Chen system (4) is the response system.

$$
\left\{\begin{array}{l}
\dot{x}_{1}=a_{1}\left(y_{1}-x_{1}\right)+u_{1} \\
\dot{y}_{1}=\left(c_{1}-a_{1}\right) x_{1}-x_{1} z_{1}+c_{1} y_{1}+u_{2} \\
\dot{z}_{1}=x_{1} y_{1}-b_{1} z_{1}+u_{3}
\end{array}\right.
$$

We add (2) from Equation (4) and yield

$$
\left\{\begin{array}{l}
\dot{e}_{1}=a_{1}\left(y_{1}-x_{1}\right)+y+u_{1} \\
\dot{e}_{2}=\left(c_{1}-a_{1}\right) x_{1}-x_{1} z_{1}+c_{1} y_{1}+z+u_{2} \\
\dot{e}_{3}=x_{1} y_{1}-b_{1} z_{1}+a x+b y+c z+x^{2}+u_{3}
\end{array}\right.
$$

Our goal is to find the proper controllers $u_{i}(i=1,2,3)$ and parameter update laws, such that system (4) globally anti-synchronizes system (2) asymptotically. i.e. 


$$
\lim _{t \rightarrow \infty}\|\mathbf{e}\|=0
$$

where $\mathbf{e}=\left[e_{1}, e_{2}, e_{3}\right]^{\mathrm{T}}$.

Theorem: If the controllers are chosen as

$$
\left\{\begin{array}{l}
u_{1}=-\hat{a}_{1}\left(x_{1}-y_{1}\right)-y-k_{1} e_{1} \\
u_{2}=-\left(\hat{c}_{1}-\hat{a}_{1}\right) x_{1}+x_{1} z_{1}-\hat{c}_{1} y_{1}-z-k_{2} e_{2} \\
u_{3}=-x_{1} y_{1}+\hat{b}_{1} z_{1}-\hat{a} x-\hat{b} y-\hat{c} z-x^{2}-k_{3} e_{3}
\end{array}\right.
$$

and the update laws of parameters are chosen as

$$
\left\{\begin{array}{l}
\dot{\hat{a}}=x e_{3} \\
\dot{\hat{b}}=y e_{3} \\
\dot{\hat{c}}=z e_{3} \\
\dot{\hat{a}}_{1}=\left(y_{1}-x_{1}\right) e_{1}-x_{1} e_{2} \\
\dot{\hat{b}}_{1}=-z_{1} e_{3} \\
\dot{\hat{c}}_{1}=\left(x_{1}+y_{1}\right) e_{2}
\end{array}\right.
$$

Then system (4) globally anti-synchronizes system (2) asymptotically, where $k_{i}(i=1,2,3)$ are positive constants, $\hat{a}, \hat{b}, \hat{c}, \hat{a}_{1}, \hat{b}_{1}, \hat{c}_{1}$ are estimate values of $a, b, c, a_{1}, b_{1}, c_{1}$, respectively.

Proof: Applying control laws (6) to (5) yields the resulting error dynamics as follows.

$$
\left\{\begin{array}{l}
\dot{e}_{1}=\tilde{a}_{1}\left(x_{1}-y_{1}\right)-k_{1} e_{1} \\
\dot{e}_{2}=\tilde{a}_{1} x_{1}-\tilde{c}_{1}\left(x_{1}+y_{1}\right)-k_{2} e_{2} \\
\dot{e}_{3}=-\tilde{a} x-\tilde{b} y-\tilde{c} z+\tilde{b}_{1} z_{1}-k_{3} e_{3}
\end{array}\right.
$$

where $\tilde{a}=\hat{a}-a, \tilde{b}=\hat{b}-b, \tilde{c}=\hat{c}-c, \tilde{a}_{1}=\hat{a}_{1}-a_{1}, \quad \tilde{b}_{1}=\hat{b}_{1}-b_{1}, \tilde{c}_{1}=\hat{c}_{1}-c_{1}$.

Consider the following Lyapunov function

$$
V=\frac{1}{2}\left(e^{\mathrm{T}} e+\tilde{a}^{2}+\tilde{b}^{2}+\tilde{c}^{2}+\tilde{a}_{1}^{2}+\tilde{b}_{1}^{2}+\tilde{c}_{1}^{2}\right)
$$

The time derivative of $V$ along the solution of error dynamical system (8) gives that

$$
\begin{aligned}
\dot{V}= & e^{\mathrm{T}} \dot{e}+\tilde{a} \tilde{\tilde{a}}+\tilde{b} \tilde{\tilde{b}}+\tilde{c} \dot{\tilde{c}}+\tilde{a}_{1} \dot{\tilde{a}}_{1}+\tilde{b}_{1} \dot{\tilde{b}}_{1}+\tilde{c}_{1} \dot{\tilde{c}}_{1} \\
= & e_{1}\left(\tilde{a}_{1} x_{1}-\tilde{a}_{1} y_{1}-k_{1} e_{1}\right)+e_{2}\left(\tilde{a}_{1} x_{1}-\tilde{c}_{1} x_{1}-\tilde{c}_{1} y_{1}-k_{2} e_{2}\right) \\
& +e_{3}\left(-\tilde{a} x-\tilde{b} y-\tilde{c} z+\tilde{b}_{1} z_{1}-k_{3} e_{3}\right)+\tilde{a}\left(x e_{3}\right)+\tilde{b}\left(y e_{3}\right) \\
& +\tilde{c}\left(z e_{3}\right)+\tilde{a}_{1}\left(\left(y_{1}-x_{1}\right) e_{1}-x_{1} e_{2}\right)+\tilde{b}_{1}\left(-z_{1} e_{3}\right)+\tilde{c}_{1}\left(x_{1}+y_{1}\right) e_{2} \\
= & -k_{1} e_{1}^{2}-k_{2} e_{2}^{2}-k_{3} e_{3}^{2}=-e^{\mathrm{T}} P e \leq 0
\end{aligned}
$$

where $P=\operatorname{diag}\left\{k_{1}, k_{2}, k_{3}\right\}$.

Since $V$ is positive definite and $\dot{V}$ is negative semi-definite in the neighborhood of zero solution of system (5), it follows that $e_{1}, e_{2}, e_{3} \in L_{\infty}, \tilde{a}, \tilde{b}, \tilde{c} \in L_{\infty}$, and $\tilde{a}_{1}, \tilde{b}_{1}, \tilde{c}_{1} \in L_{\infty}$, from (8), we have $\dot{e}_{1}, \dot{e}_{2}, \dot{e}_{3} \in L_{\infty}$, since $\dot{V}=-e^{\mathrm{T}} P e$, we obtain

$$
\int_{0}^{t} \lambda_{\min }(P)\|e\|^{2} \mathrm{~d} t \leq \int_{0}^{t} e^{\mathrm{T}} P e \mathrm{~d} t=\int_{0}^{t}-\dot{V} \mathrm{~d} t=V(0)-V(t) \leq V(0)
$$

where $\lambda_{\min }(P)$ is the minimal eigenvalue of the positive definite matrix $P$. Thus, $\dot{e}_{1}, \dot{e}_{2}, \dot{e}_{3} \in L_{2}$, by Barbalat's lemma, we have $\lim _{t \rightarrow \infty}\|e(t)\|=0$. Therefore, response system (4) can globally anti-synchronize drive system (2) asymptotically. This completes the proof. 
In simulation, Fourth order Runge-Kutta integration method is used to solve the systems of differential Equations (2) and (4) with the controllers (6) and the parameter update laws (7). We select the parameters of Genesio system as $a=-6, b=-2.92, c=-1.2$ and the parameters of Chen system as $a_{1}=35, b_{1}=3, c_{1}=28$, respectively, and $k_{i}(i=1,2,3)=1$. The initial values of drive and response systems are $x(0)=0.2, y(0)=0.4$, $z(0)=0.6$ and $x_{1}(0)=-2, y_{1}(0)=2, z_{1}(0)=5$, respectively, while the initial errors of system (5) are $e_{1}(0)=-1.8, e_{2}(0)=2.4, e_{3}(0)=5.6$ and the initial values of estimate parameters are $\hat{a}(0)=\hat{b}(0)=\hat{c}(0)=1$, $\hat{a}_{1}(0)=\hat{b}_{1}(0)=\hat{c}_{1}(0)=2$. The anti-synchronization errors between Chen system and Genesio system are shown in Figure 1, the estimate values of parameters $a, b, c$ and $a_{1}, b_{1}, c_{1}$ are shown in Figure 2 and Figure 3, respectively. Obviously, the anti-synchronization errors converge asymptotically to zero and two different systems are indeed achieved chaos anti-synchronization. Furthermore, the estimates of parameter converge to their true values.
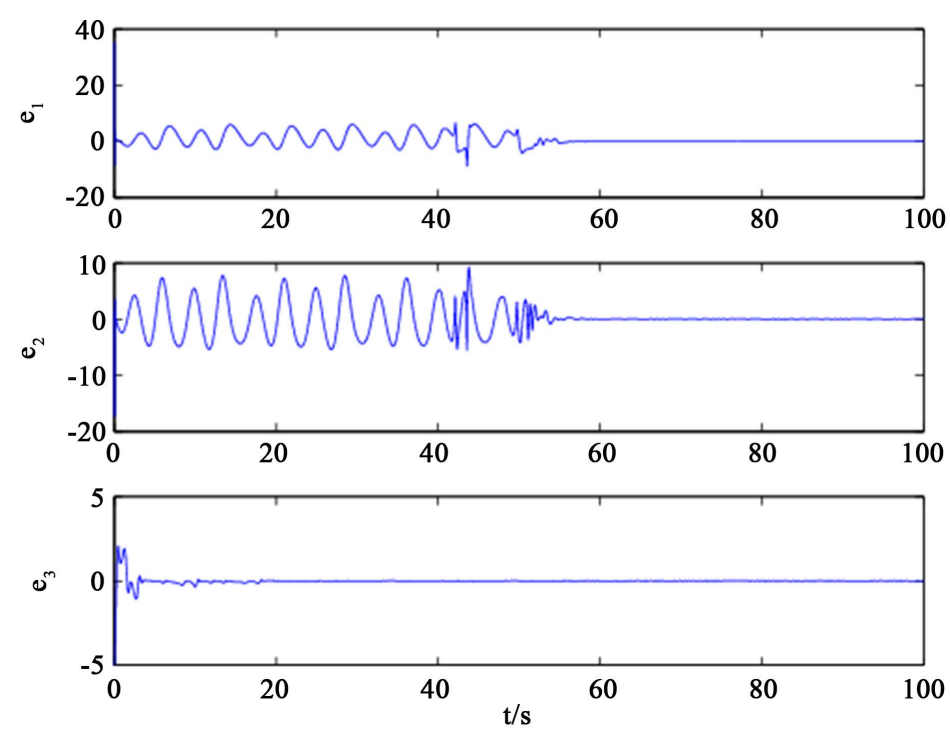

Figure 1. Anti-synchronization errors between Chen and Genesio systems via adaptive control.
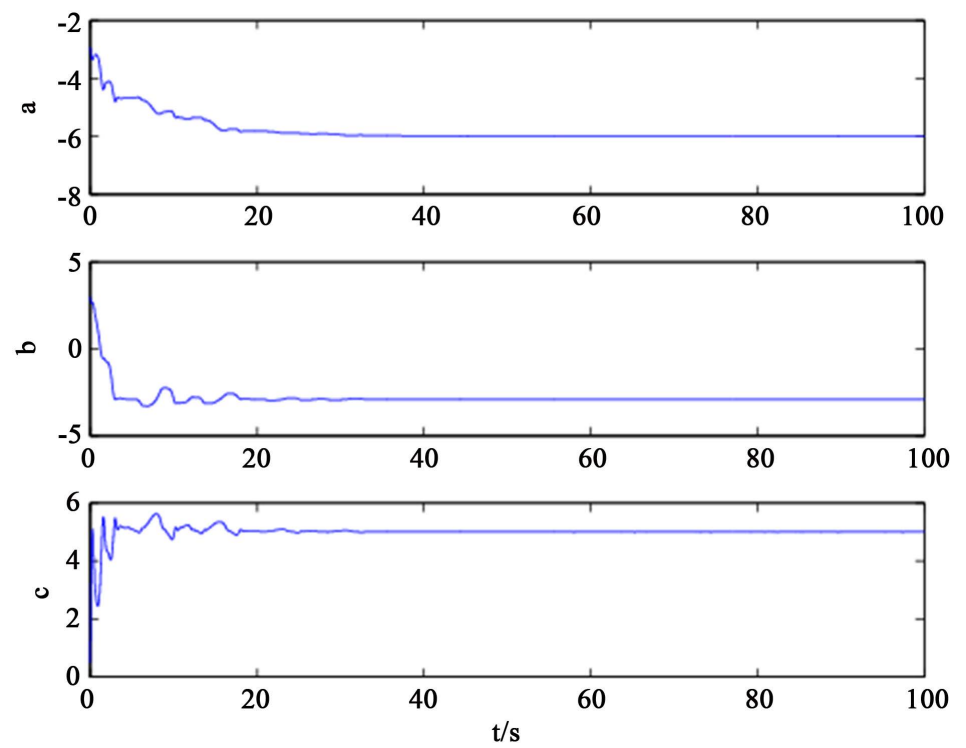

Figure 2. Estimate values of parameters $a, b, c$ of drive system. 

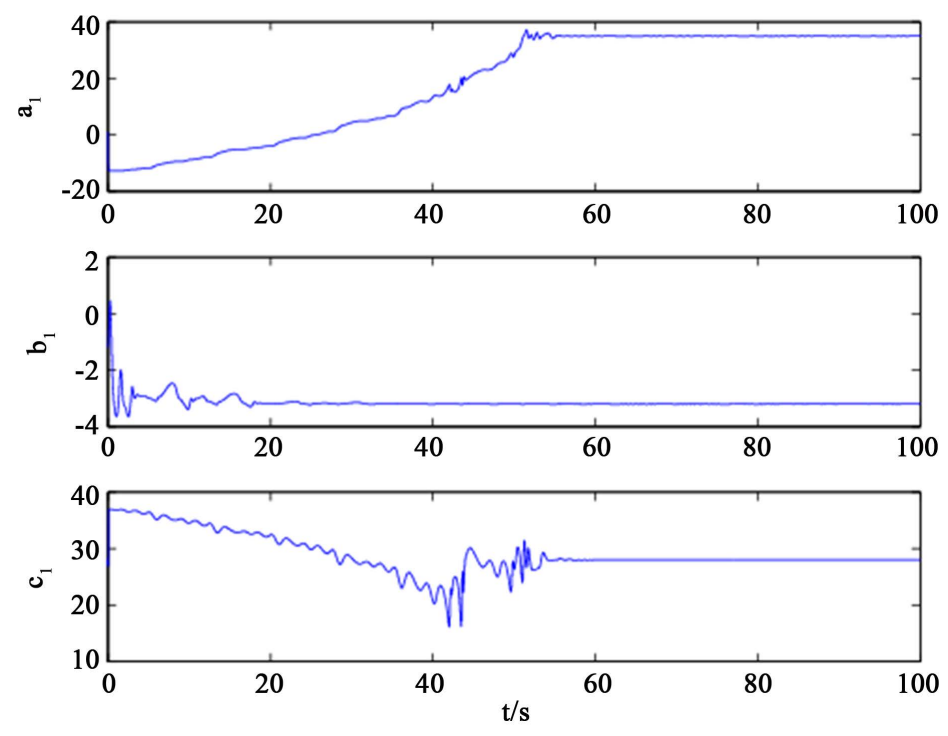

Figure 3. Estimate values of parameters $a_{1}, b_{1}, c_{1}$ of response system.

\section{Conclusion}

In this paper, chaos anti-synchronization between two different chaotic systems with different structures using adaptive control is presented. Chen system and Genesio system are taken as an illustrative example to verify the effectiveness of the proposed methods.

\section{References}

[1] Pecora, L.M. and Carroll, T.L. (1990) Synchronization in Chaotic Systems. Physical Review Letters, 64, 821-824. http://dx.doi.org/10.1103/PhysRevLett.64.821

[2] Kocarev, L. and Parlitz, U. (1996) Generalized Synchronization, Predictability, and Equivalence of Unidirectional Coupled Dynamical Systems. Physical Review Letters, 76, 1816-1819. http://dx.doi.org/10.1103/PhysRevLett.76.1816

[3] He, X., Li, C.D., Huang, J.J. and Xiao, L. (2015) Generalized Synchronization of Arbitrary-Dimensional Chaotic Systems. Optik, 126, 454-459. http://dx.doi.org/10.1016/j.ijleo.2014.09.005

[4] Koronovskii, A.A., Moskalenko, O.I., Ponomarenko, V.I., et al. (2016) Binary Generalized Synchronization. Chaos, Solitons and Fractals, 83, 133-139. http://dx.doi.org/10.1016/j.chaos.2015.11.045

[5] Radhakrishnan, S., Duvvuru, A., Sultornsanee, S. and Kamarthi, S. (2016) Phase Synchronization Based Minimum Spanning Trees for Analysis of Financial Time Series with Nonlinear Correlations. Physica, 444, 259-270. http://dx.doi.org/10.1016/j.physa.2015.09.070

[6] Ma, Y. and Zheng, Y. (2015) Projective Lag Synchronization of Markovian Jumping Neural Networks with Mode-Dependent Mixed Time-Delays Based on an Integral Sliding Mode Controller. Neurocomputing, 168, 626-636. http://dx.doi.org/10.1016/j.neucom.2015.05.062

[7] Li, W.L., Chen, X.Q. and Shen, Z.P. (2008) Anti-Synchronization of Two Different Chaotic Systems. Physica A, 387, 3747-3750. http://dx.doi.org/10.1016/j.physa.2008.02.011

[8] Fu, G.Y. and Li, Z.S. (2011) Robust Adaptive Anti-Synchronization of Two Different Hyperchaotic Systems with External Uncertainties. Communications in Nonlinear Science and Numerical Simulation, 16, 395-401. http://dx.doi.org/10.1016/j.cnsns.2010.05.015

[9] Mossa Al-sawalha, M., Noorani, M.S.M. and Al-Dlalah. M.M. (2010) Adaptive Anti-Synchronization of Chaotic Systems with Fully Unknown Parameters. Computers and Mathematics with Applications, 59, 3234-3244. http://dx.doi.org/10.1016/j.camwa.2010.03.010

[10] Singh, P.P., Singh, J.P. and Roy, B.K. (2014) Synchronization and Anti-Synchronization of Lu and Bhalekar-Gejji Chaotic Systems Using Nonlinear Active Control. Chaos, Solitons \& Fractals, 69, 31-39. http://dx.doi.org/10.1016/j.chaos.2014.09.005

[11] Genesio, R. and Tesi, A. (1992) Harmonic Balance Methods for the Analysis of Chaotic Dynamics in Nonlinear Systems. Automatica, 28, 531-548. http://dx.doi.org/10.1016/0005-1098(92)90177-H 
Submit or recommend next manuscript to OALib Journal and we will provide best service for you:

- Publication frequency: Monthly

- 9 subject areas of science, technology and medicine

- Fair and rigorous peer-review system

- Fast publication process

- Article promotion in various social networking sites (LinkedIn, Facebook, Twitter, etc.)

- Maximum dissemination of your research work

Submit Your Paper Online: Click Here to Submit

Contact Us: service@oalib.com 